\title{
2017;9(1):53-60. DOI: 10.18869/acadpub.ijwph.9.1.5. \\ Prevalence of Physical Diseases and Disorders in Retired Armed Forces; a Case Study of Tehran City, Iran
}

\section{ART ICLE INF O}

\section{Article Type}

Descriptive Study

\section{Authors}

Pirooz F.* MD, $M P H$,

Mohammadi Shahbolaghi F. ${ }^{1} P h D$, Foroughan M. ${ }^{1} M D$

\begin{tabular}{l} 
How to cite this article \\
\hline Pirooz F, Mohammadi Shahbola- \\
ghi F, Foroughan M. Prevalence of \\
Physical Diseases \& Disorders in \\
Retired Armed Forces; a Case St- \\
udy of Tehran City, Iran. Iranian \\
Journal of War \& Public Health. \\
2017;9(1):53-60.
\end{tabular}

*Iranian Research Center on Ageing, University of Welfare and Rehabilitation Sciences, Tehran, Iran ${ }^{1}$ Iranian Research Center on Ageing, University of Welfare and Rehabilitation Sciences, Tehran, Iran

\section{Correspondence}

Address: Iranian Research Center on Ageing, University of Social Welfare and Rehabilitation Sciences, Koodakyar Street, Daneshjoo Buolvarde, Evin, Tehran, Iran

Phone: +98 (21) 22548008

Fax: +98 (21) 22546043

fereshtehpirooz@gmail.com

\section{Article History}

Received: October 13, 2016

Accepted: January 7, 2017

ePublished: March 10, 2017

\section{A B S T RA C T}

Aims The first step to respond to the health needs of the retirees of the military organizations is to identify such needs. The aim of the study was to determine the frequencies of physical problems and diseases of the military retirees, as well as to determine the potential impact of the services provided by the military organiztions on the old-age health needs.

Instrument \& Methods In the cross-sectional descriptive-analytic study, 299 military retirees, who were residing in Tehran, were studied between 2013 and 2015. The subjects were selected via available purposeful sampling method method. Data was collected by the study questionnaire consisted of the abbreviated mental test score (AMTS) and a researchermade questionnaire as the main survey of the study. Data was analyzed by SPSS 16 software using descriptive statistics and one-way ANOVA and independent T tests.

Findings 159 retirees (\%53.2) were without any communication-limiting physical problem. Nevertheless, the most prevalent limiting disorder was the auditory problems (12.0\%). 144 retirees $(48.2 \%)$ were with active diseases, and the most prevalent diseases were high blood pressure $(21.5 \%)$ and diabetes (19.4\%). Totally, the physical health status was declared by 214 retirees $(71.6 \%)$, and 15 retirees $(5.0 \%), 77$ retirees $(25.7 \%)$, and 99 retirees (33.1\%) declared their own satisfaction levels as very good, good, and moderate, respectively.

Conclusion The most important health problems of the military retirees are the chronic noncommunicable diseases such as high blood pressure, diabetes, and cardio-vascular diseases. Only one third of the subjects are satisfied with their own health status.

\section{Keywords Disease; Retirement; Armed Forces Personnel}

\section{I T A T I O N L I N KS}

[1] Community mobilization to promote the health of the elderly in the neighborhood Complex ... [2] The global economic burden of non-communicable diseases ... [3] Comparing quality of life between seniors living in families ... [4] Health-related quality of life in an elderly population in Iran: A population... [5] A Demographic characteristics of the elderly population in Iran according to the census ... [6] Assess the health needs aged pensioners of social security organization ... [7] Civic Engagement among older Chinese internet ... [8] Military deployments and children's academic achievement: Evidence from department of defense education ... [9] Health of national Service Veterans: an analysis ... [10] Is the SF-36 a valid measure of change in population health? results from the whitehall ... [11] A review of our roots: Blacks in gerontology ... [12] The injured mind in the UK Armed Forces ... [13] Life-space mobility and quality of life in community ... [14] Development of a community based care system ... [15] Elderly quality of life: A comparison between pensioners of Social ... [16] Tough bodies and rough sleeping: Embodying ... [17] Factors associated with heavy alcohol consumption in the UK Armed Forces: Data from a health survey ... [18] The status of social and leisure time activities among elderly residing in Iran [19] Validation of the persian version of abbreviated mental... [20] Relationship between social participation and general health among the ... [21] Mental health condition and health promoting behaviors among elders in ... [22] Assessing the health status of elderly people in the province ... [23] What's in a name? defining and caring for ... [24] A study on the quality of life among elderly shahinshahr area of isfahan ... [25] The elderly population in Iran: An ever growing concern in ... [26] LEIPAD, an internationally applicable ... [27] Association between polypharmacy and socio-demographic ... [28] Inappropriate prescribing ... [29] Study of polypharmacy and associated problems among elderly ... [30] Recent patterns of medication use in ... [31] Attitudes toward older people among nursing students and registered nurses ... 


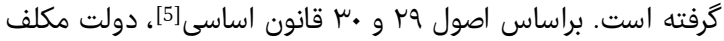

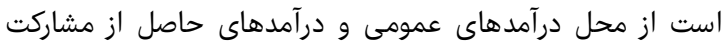

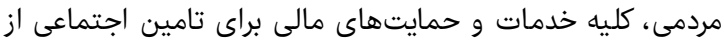

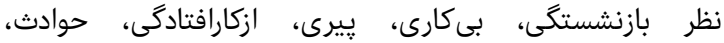

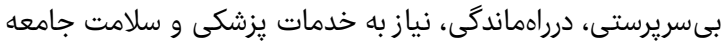

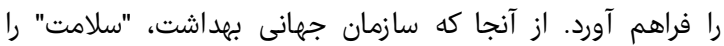

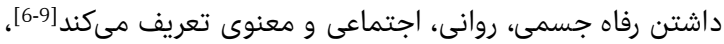

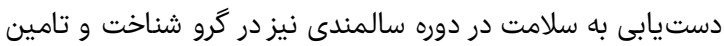

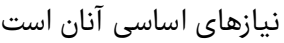

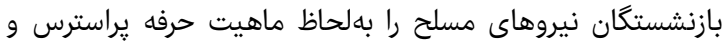

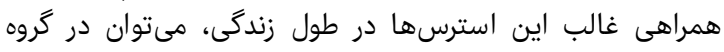

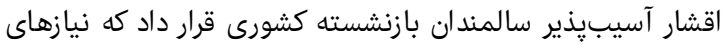

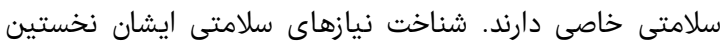

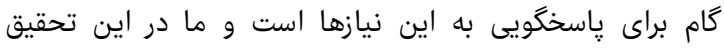

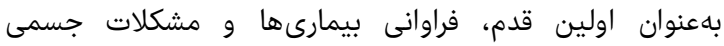

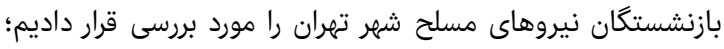

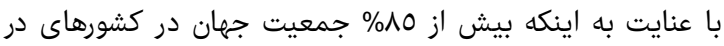

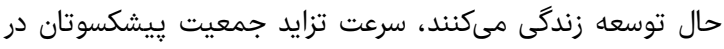

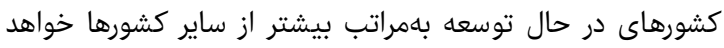

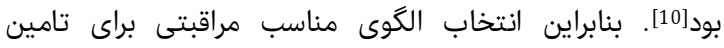

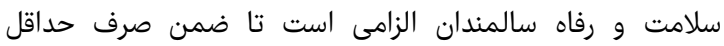

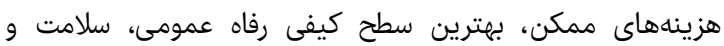

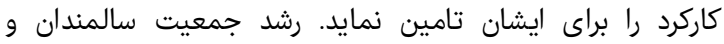

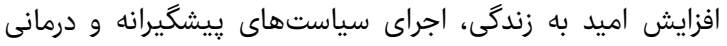
مناسب را براى حفظ و ارتقاى سطح سلامت ايشان بـان الزامى درانى

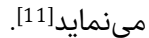

از آنجا كه در بسيارى از مناطق دنيا به دلايل ساختارى و امكان

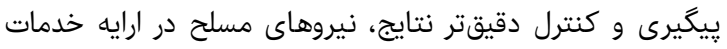

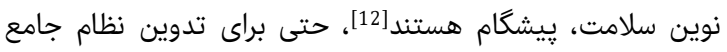

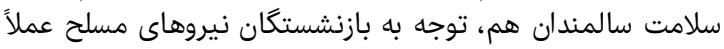

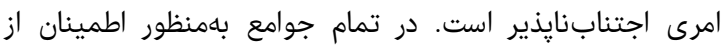

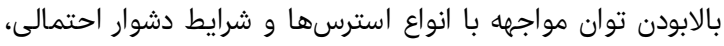

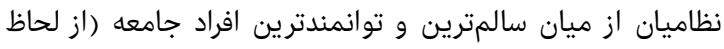

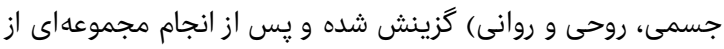

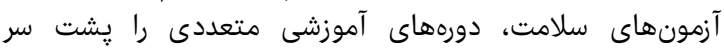

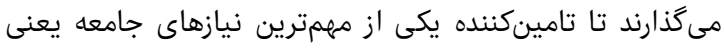

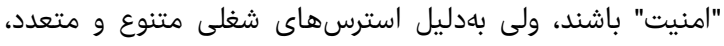

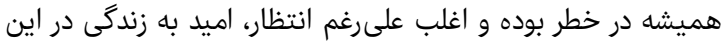

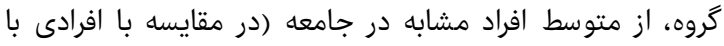

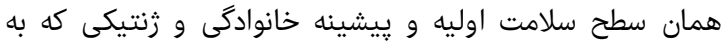

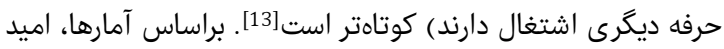

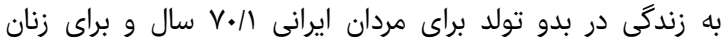

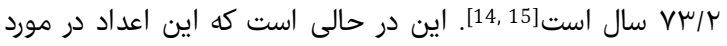

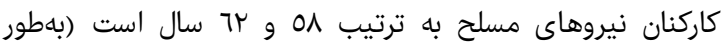

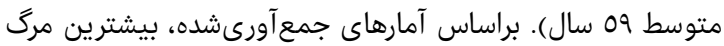

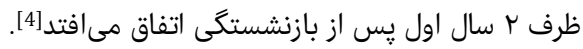

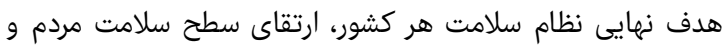

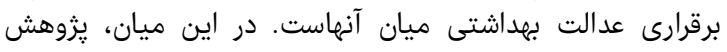

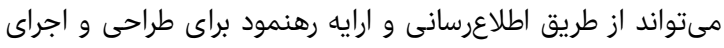

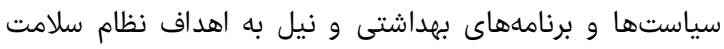

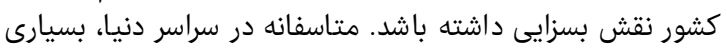

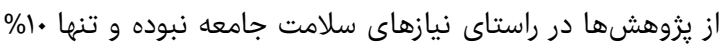

\section{فراوانى بيمارىها و و مشكلات جسمى در بازنشستكان نيروهاى مسلح؛ مطالعه موردى شهر تمهران}

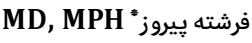 \\ مركز تحقيقات سالمندى، دانشكاه علوم بهزيستى وتوانبخشى، تهران، ايران

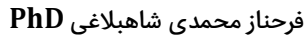

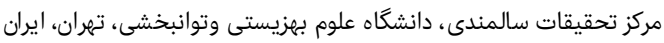 \\ MD مهشيد فروغان \\ مركز تحقيقات سالمندى، دانشكاه علوم بهزيستى وتوانبخشى، تهران، ايران
}

حكيده

اهداف: شناخت نيازهاى سلامتى بازنشستكان نيروهاى مسلح، نخستين كام إم

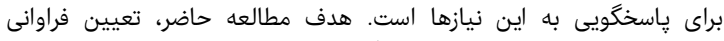

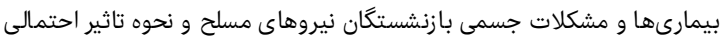

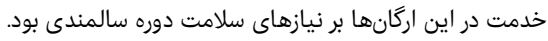

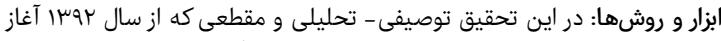

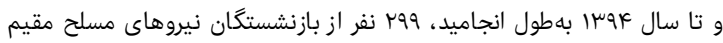

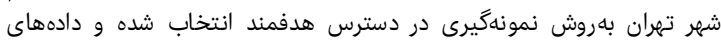

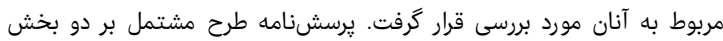

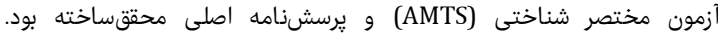

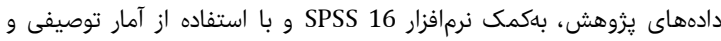

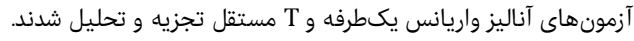

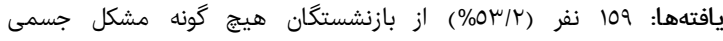

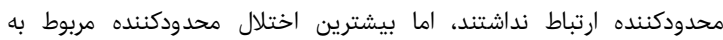

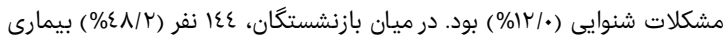

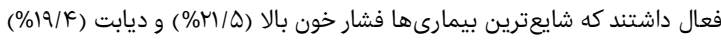

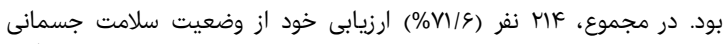

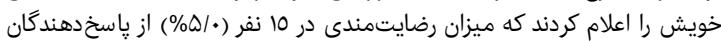

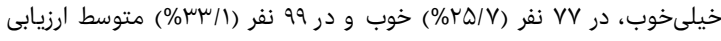

نتيجهكيرى: مهمترين مشكل سلامتى بازنشستكان نيروهاى مسلح، ابتلا به

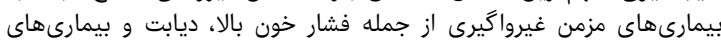

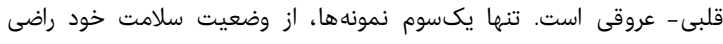

كليدواثمها: مشكلات جسمى، بيمارىها، بازنشستكان، نيروهاى مسلح

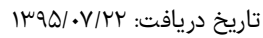

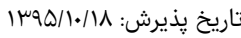

fereshtehpirooz@gmail.com : نويسنده مسئول:

مقدمه - - مقد

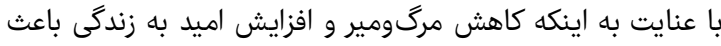

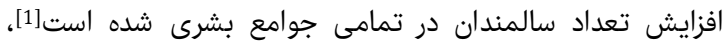

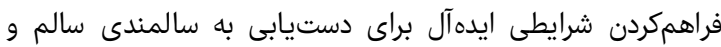

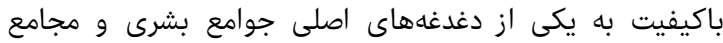

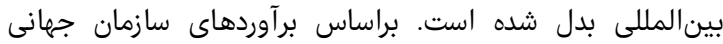

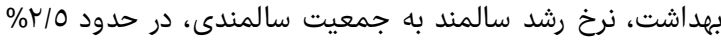

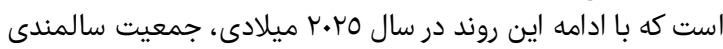

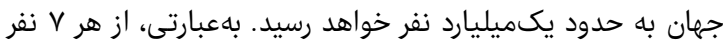

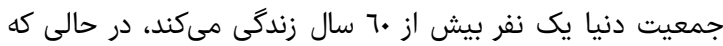

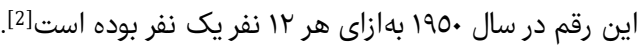

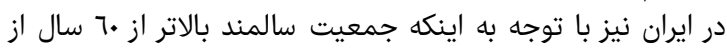

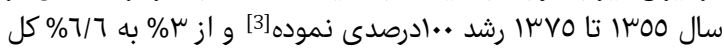

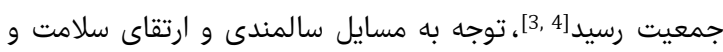

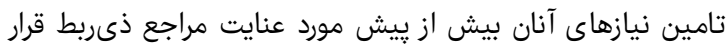




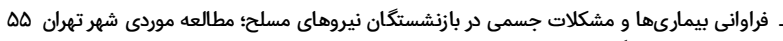

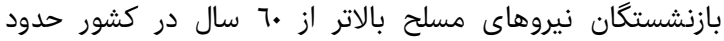

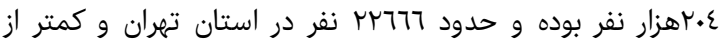

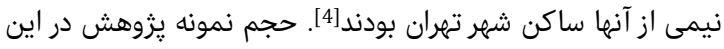

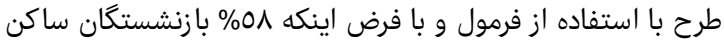

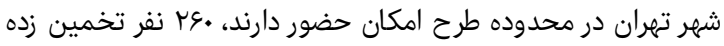

محقق از زمان كسب مجوزهاى فعاليت (سهماهه سوم سال بوسبا)،

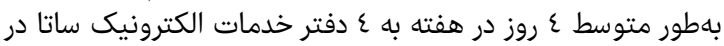

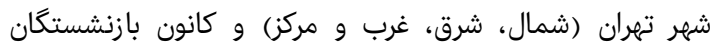

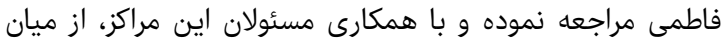

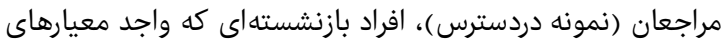

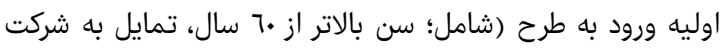

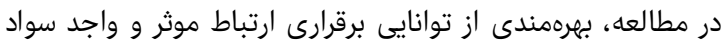

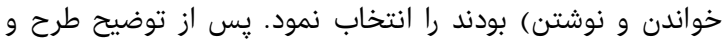

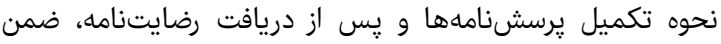

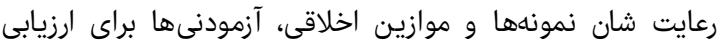

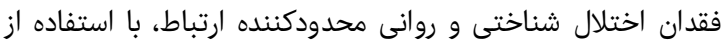

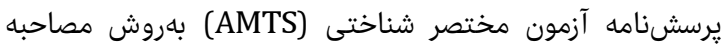

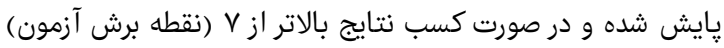

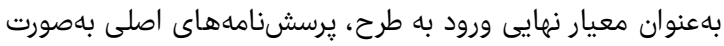

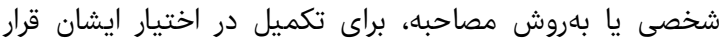

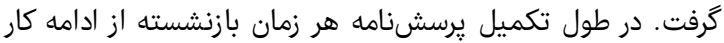

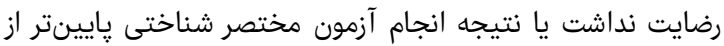

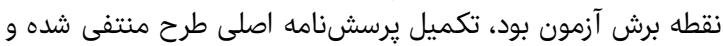

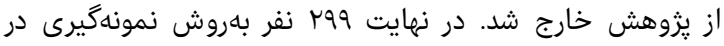

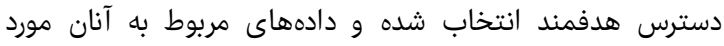
برسى قرار گرفت. يرسشنامه طرح مشتمل بر دو بخش بردي بودي يكى ارزيابى وضعيت

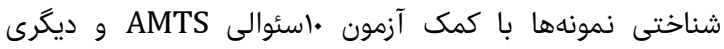

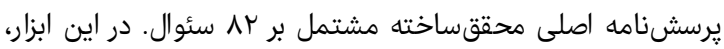

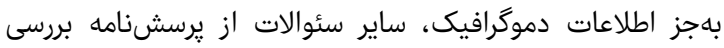

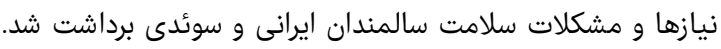

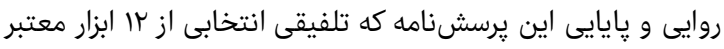

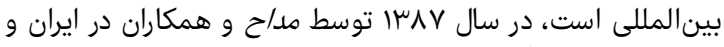

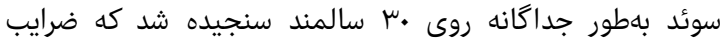

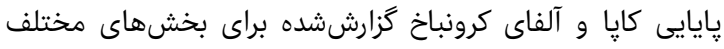

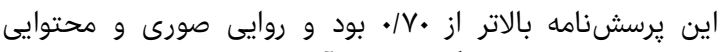

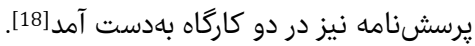

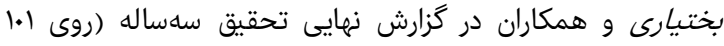

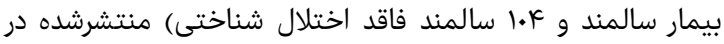

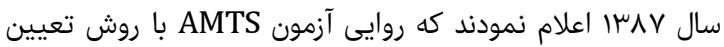

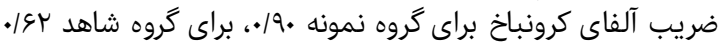

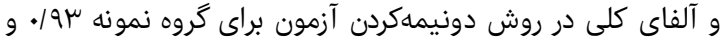

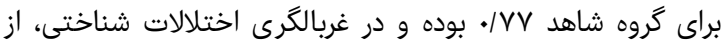

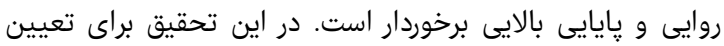

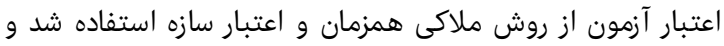

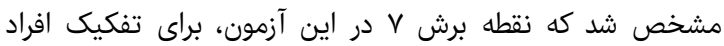

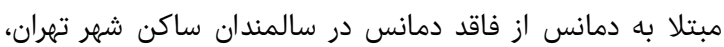

نقطه برش مطلوب است[19]. محقق در ابتدا معرفىنامههاى لازم براى حضور در دراست دفاتر و مراكز

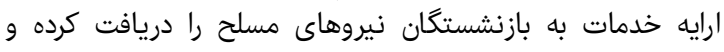

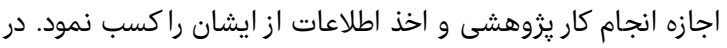

كل سرمايههاى يزوهشى دنيا براى .9\% مردم جهان بهكار

مىرود [16].

محقق و همكاران در مطالعه خود كه با هدف بررسى نيازهاى

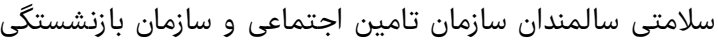

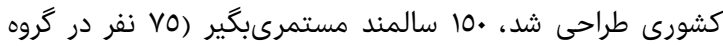

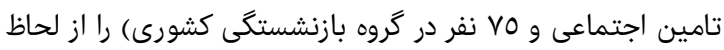

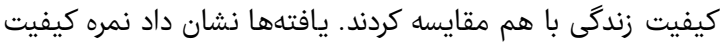

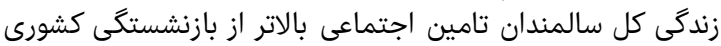

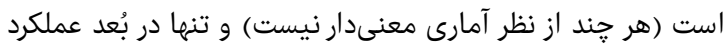

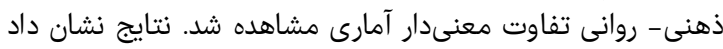

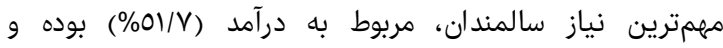

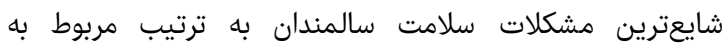

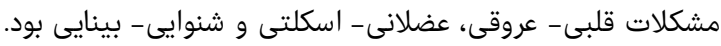

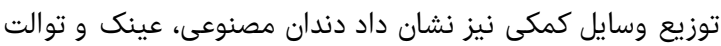

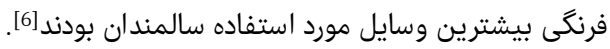

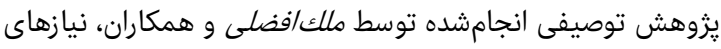

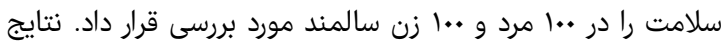

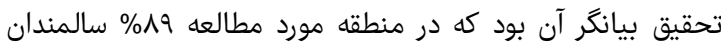

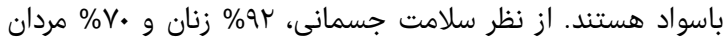

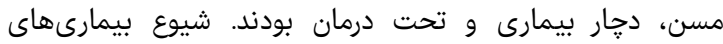

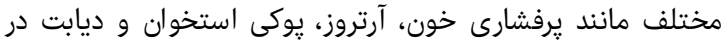

زنان بيشتر از مردان بود[1].

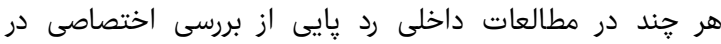

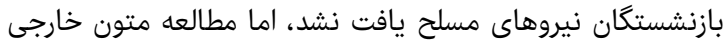

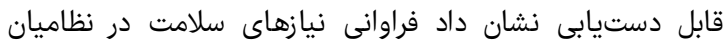

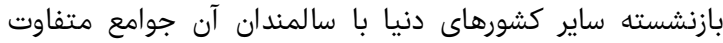

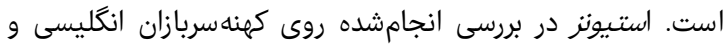

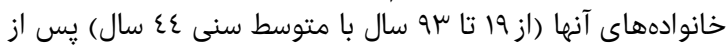

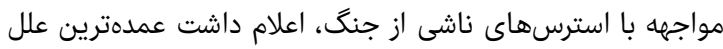

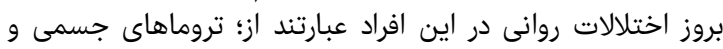

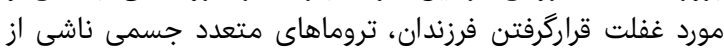

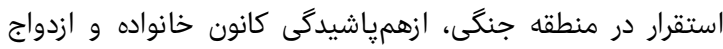

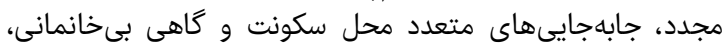

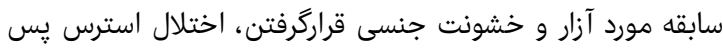

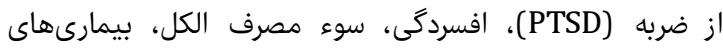

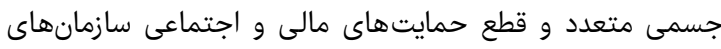

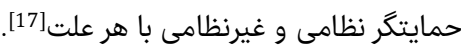

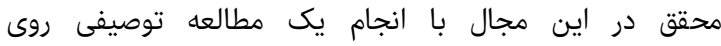

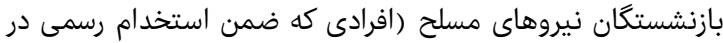

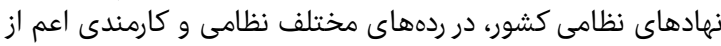
زن و مرد، واجد سوابق ادارى شامل؛ كارت، كد شناسايى و فيش فيش

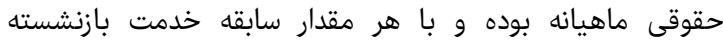

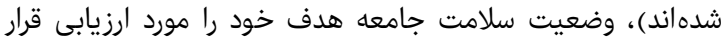

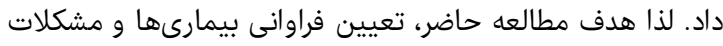

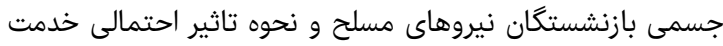

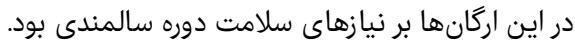

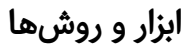

اين تحقيق توصيفى- تحليلى و مقطعى كه از سال بوسرا آغاز و تا

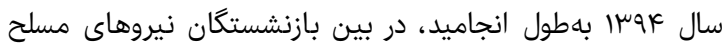

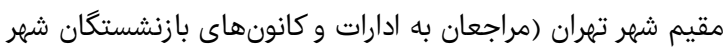

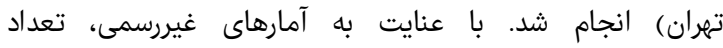




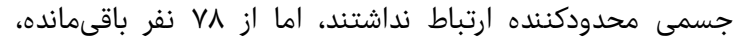

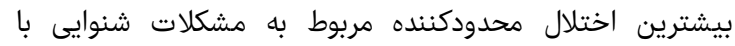

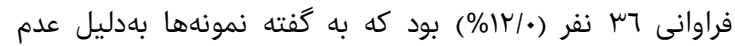

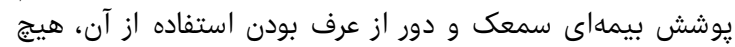

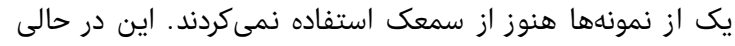

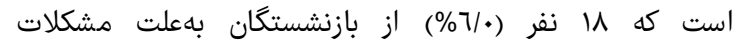

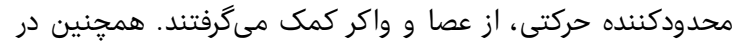

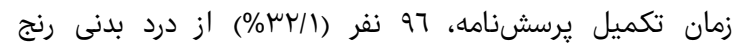

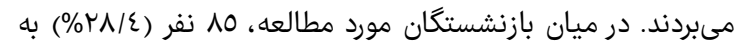

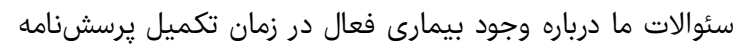

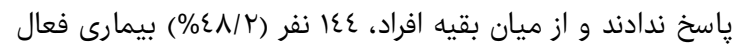

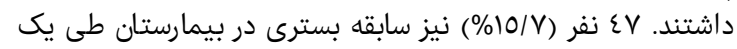

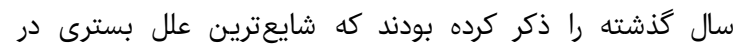

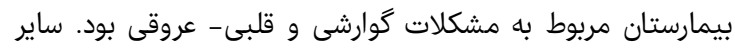

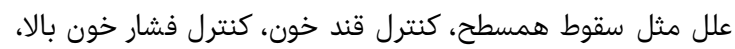

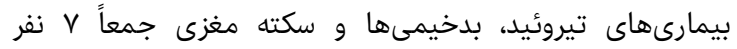

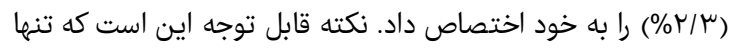

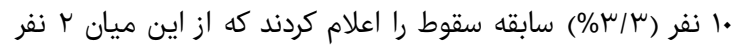

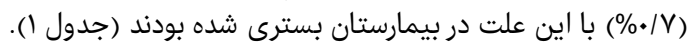

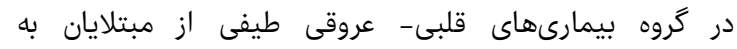

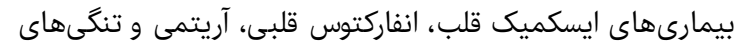

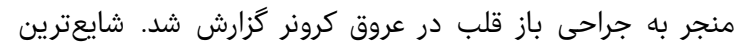

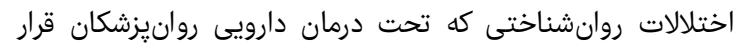

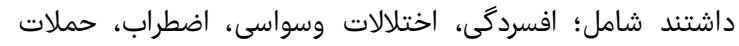

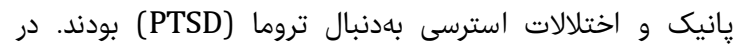

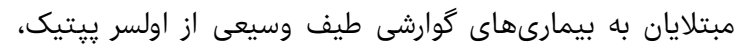

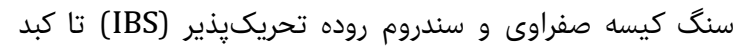

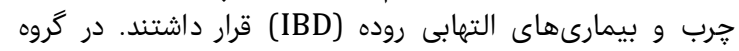

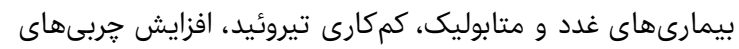

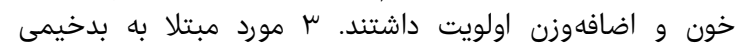

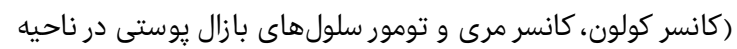

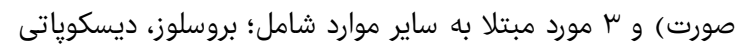

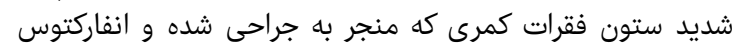

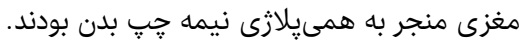

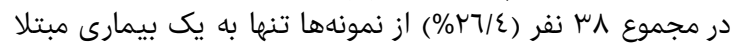

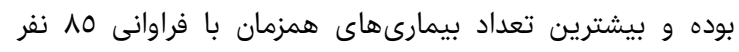

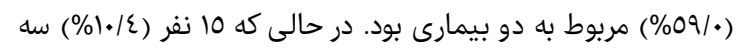

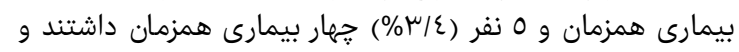

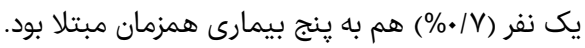

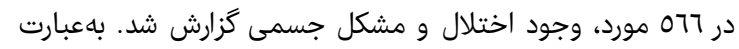

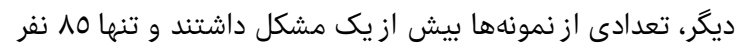

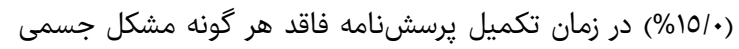

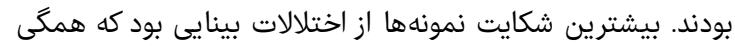

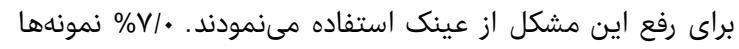

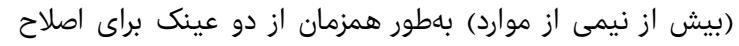

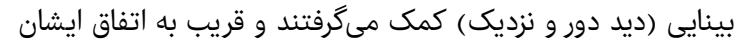

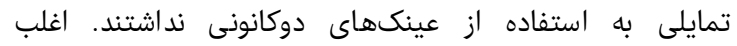

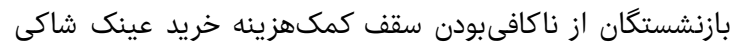

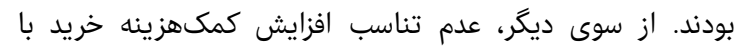

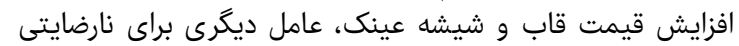

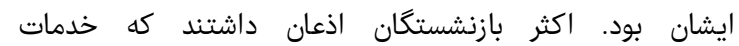
دندانيزشكى اغلب كران و محدود به مراكز خاصى بوده و توزيع
طول انجام مراحل ادارى كار، ادارات جهاركانه بازنشستخى منحل

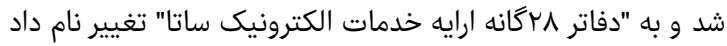

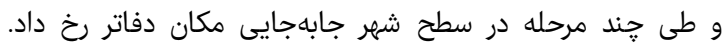

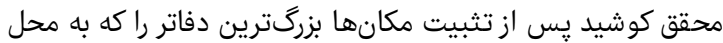

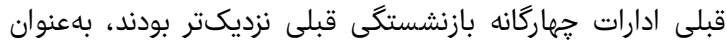

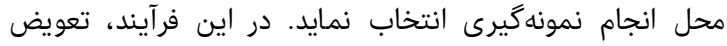

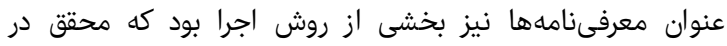

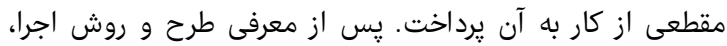

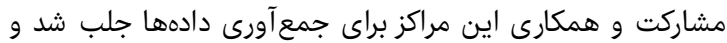

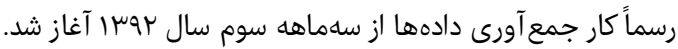

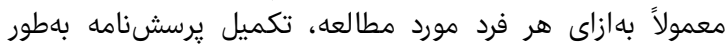

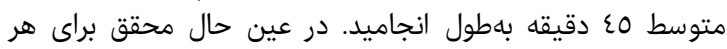

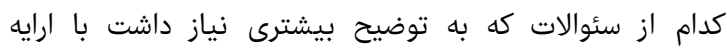

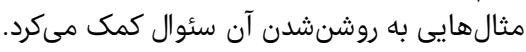

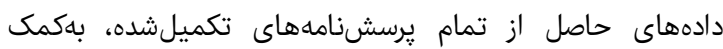

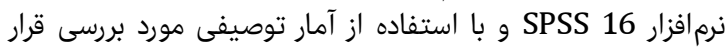

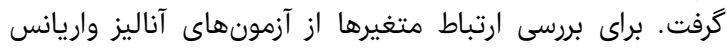
يكنرفه و T مستقل استفاده شد.

\section{يافتهها}

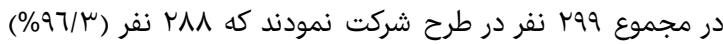

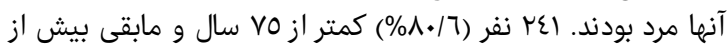

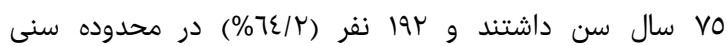

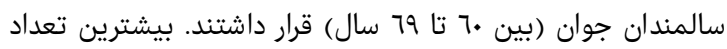

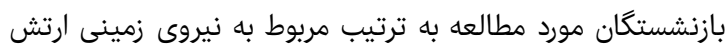

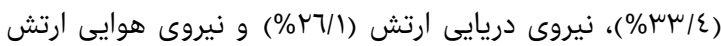

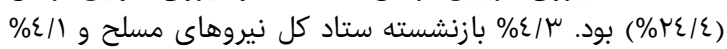

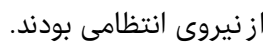

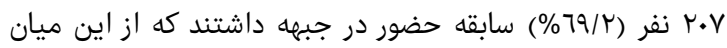

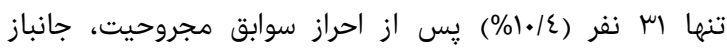

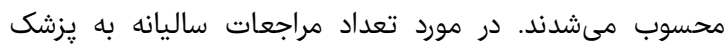

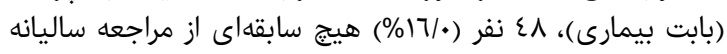

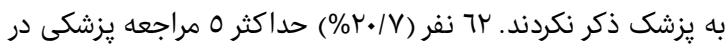

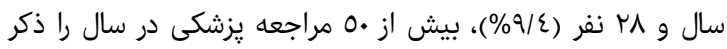
نمودند.

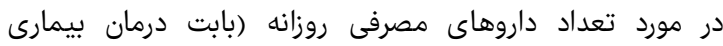

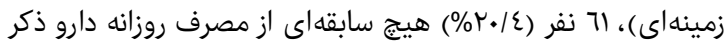

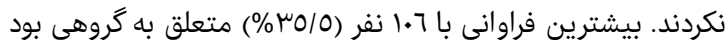

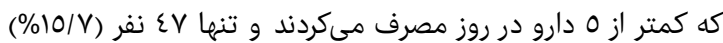

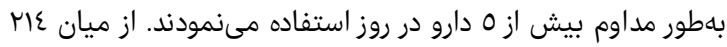

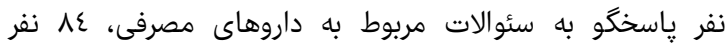

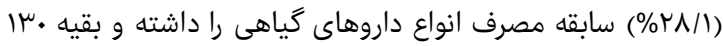

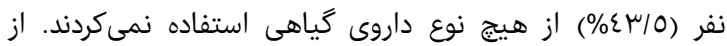

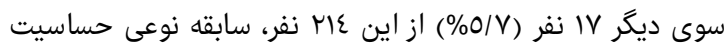

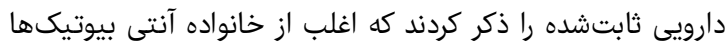

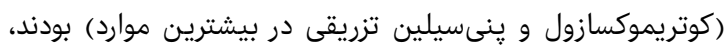

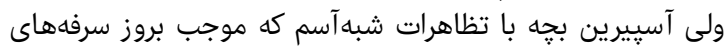

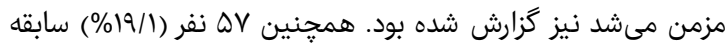

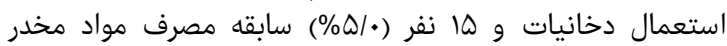

داشتند.

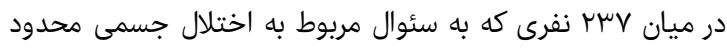

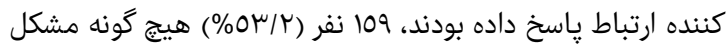




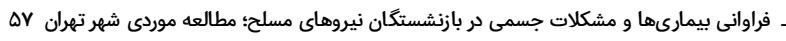

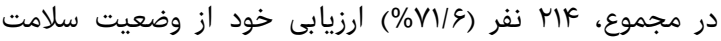

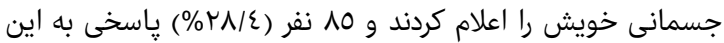

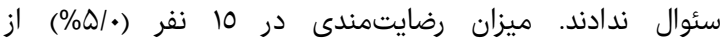

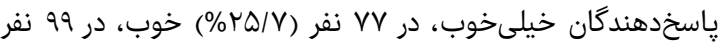
(ا/ خيلىبد ارزيابى شد. در بررسى متغيرهاى جمعيتشناختى، متغير سن با شش مولفي

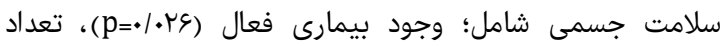

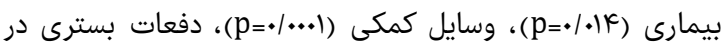
سال (pr

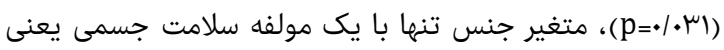

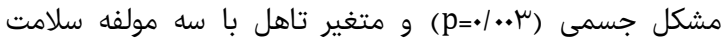

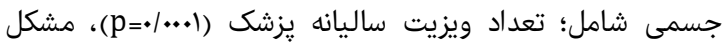

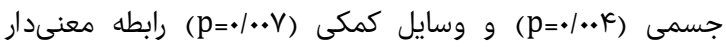
داشتند. خودارزيابى بازنشسته از وضعيت سلامت كلى با سه متغئير

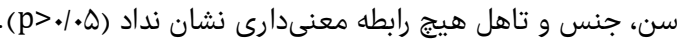

بحث

بيشتر بازنشستگان مورد مطالعه در شهر تهران، وضعيت سلامت

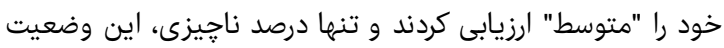

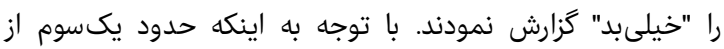

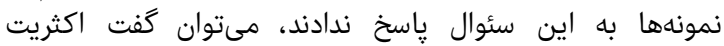

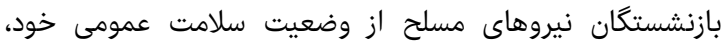
ناراضى نبودند كه اين يافته با مطالعه يزد/نى و همان هماران دران

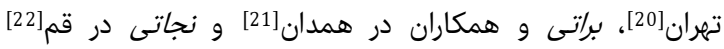
همراستا بود.

شايعترين بيمارىها در بازنشستكان نيروهاى مسلح در تهران بهان بهاري

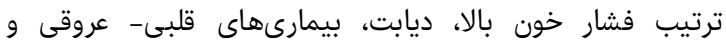

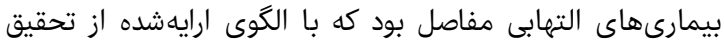

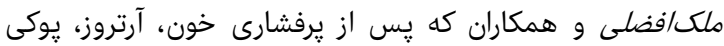

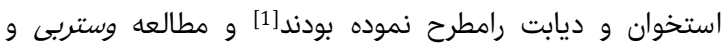

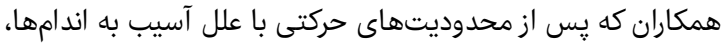

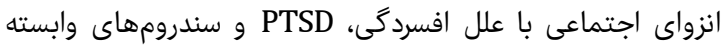

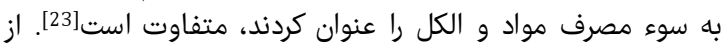

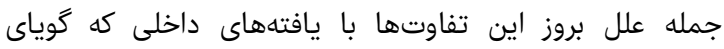

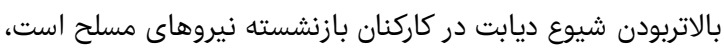

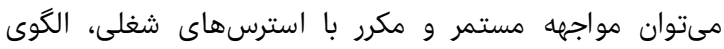

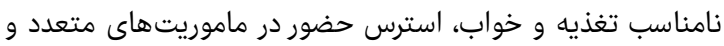

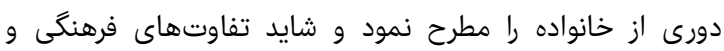

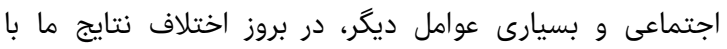
يافتهاهاى خارجى نقش دارند كه براى بررسى درى آن، به انجام مطالعات بيشترى نياز است.

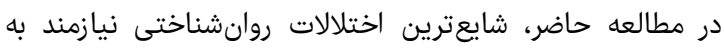
درمان دارويى روانيزشكان در بازنشستكان نيروهاى مسلح، بـانه

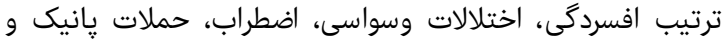
بودند كه مورد مشابه داخلى نداشته و با گزارشات وستربى

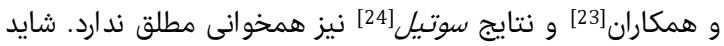
در مطالعات خارجى شيوع بالاتر سوء مصرف مواد و الكل، زندگى

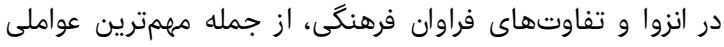
باشند كه بتوان بهعنوان علل بروز اين اختلاف در نتايج، مورد توجه

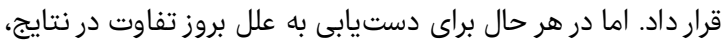

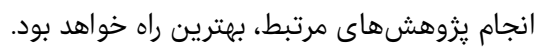

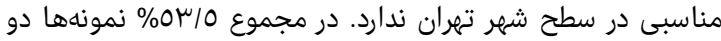

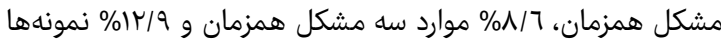
از جهار مشكل جسمى همزمان شاكى بودند.

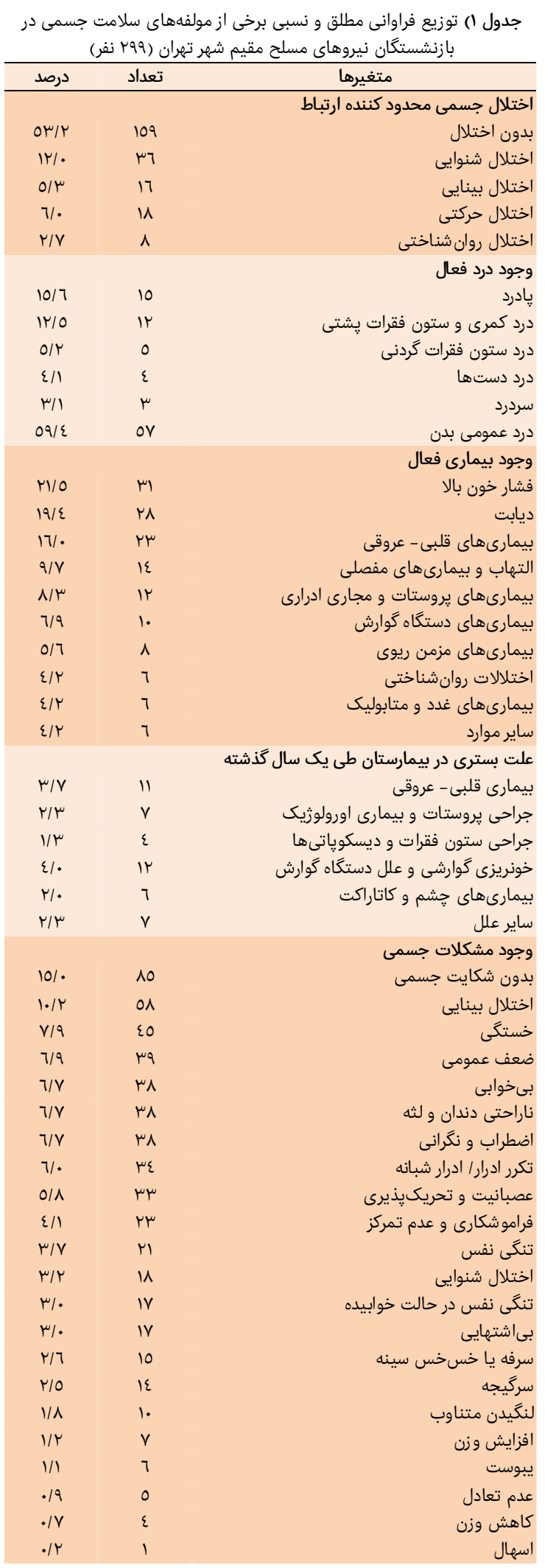

Volume 9, Issue 1, Winter 2017 
مشكلات جسمى را به خود اختصاص مىدادند. بديهى است رسالت

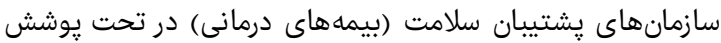

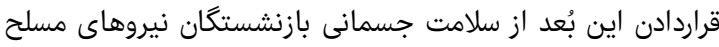

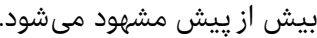
بيش از نيمى از نمونههايى كه به به سئوال ما ياسخ دادئ داده بودند، در

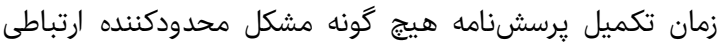

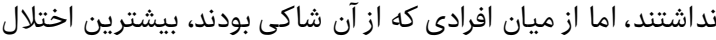

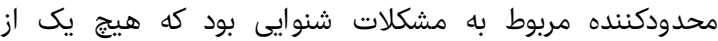

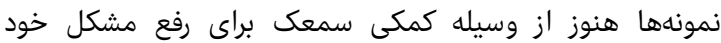

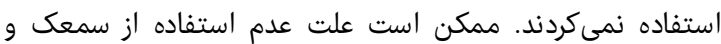

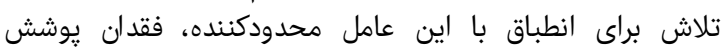

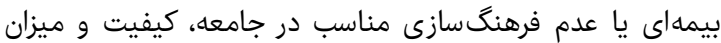

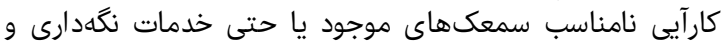

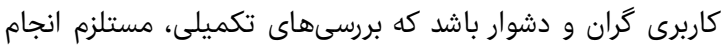

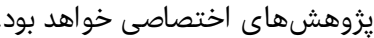

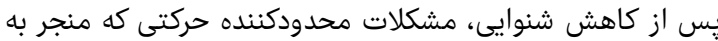

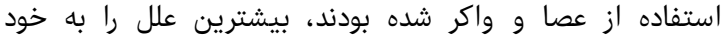

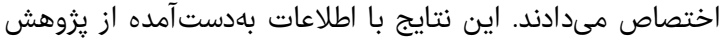

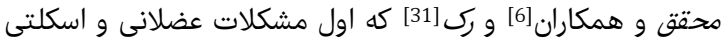

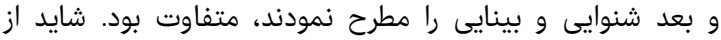

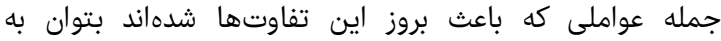

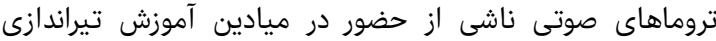

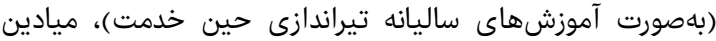

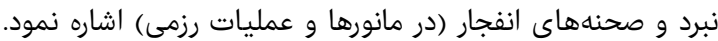

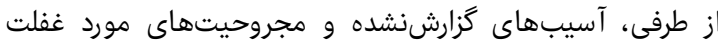

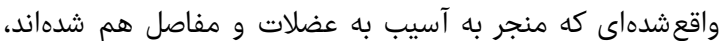

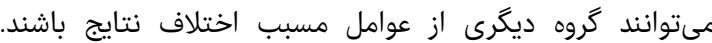

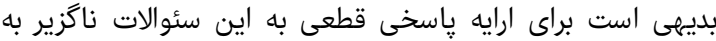

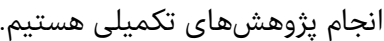

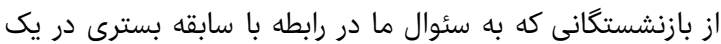

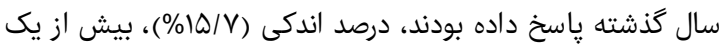

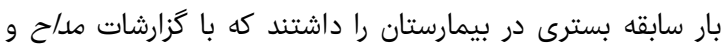

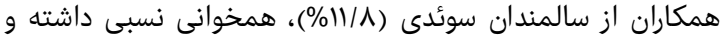

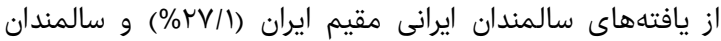

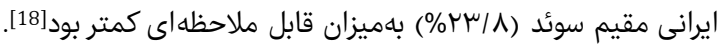

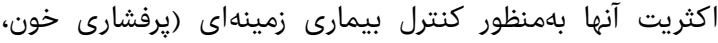

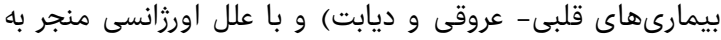

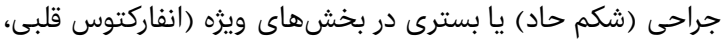

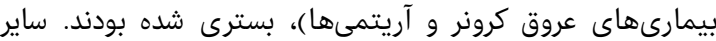

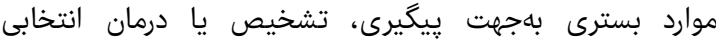

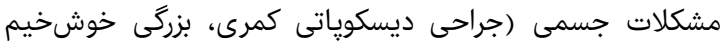

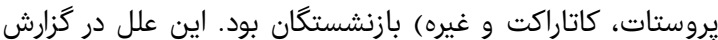

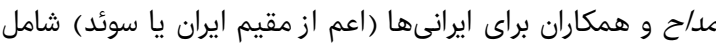

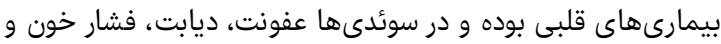

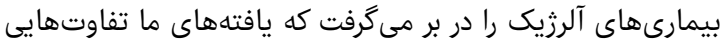

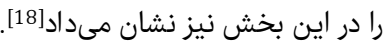

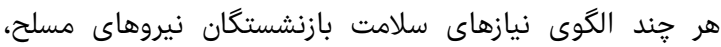

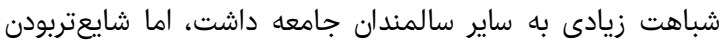

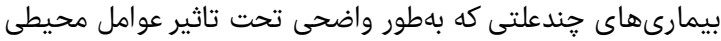

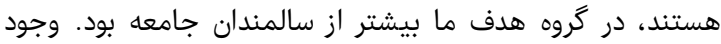

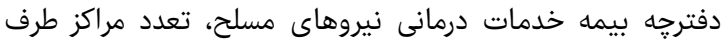

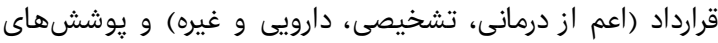

دوره 9، شماره ا، زمستان هوسر

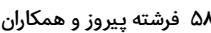
كوششى و آقايى در سال بوحسا تغييرات •r علت اصلى سالهاى

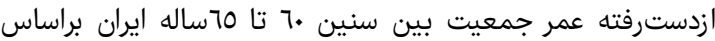

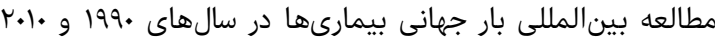

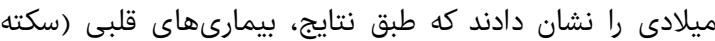

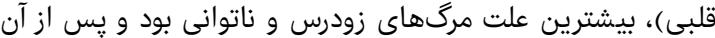

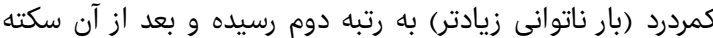

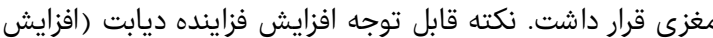

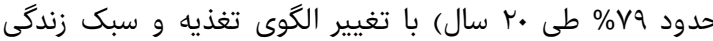

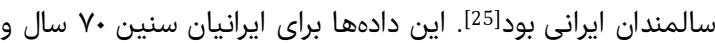

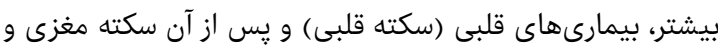

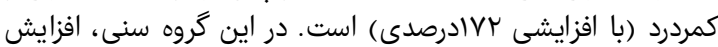

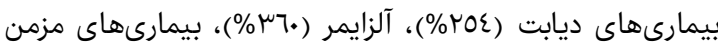

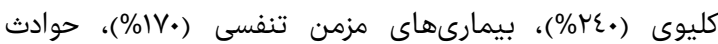

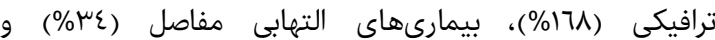

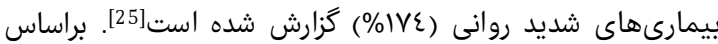

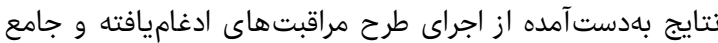

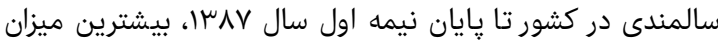

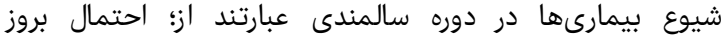

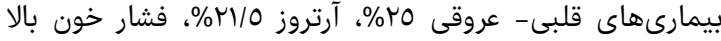

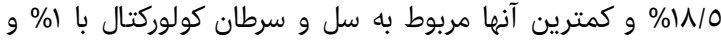

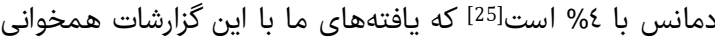

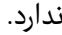
شيوع مصرف همزمان 0 يا بيش از 0 دارو در روز روز يا به تعبير

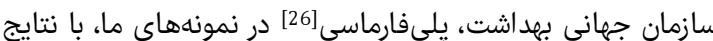

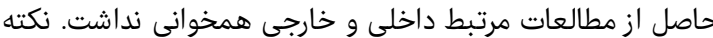

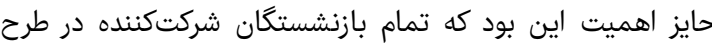

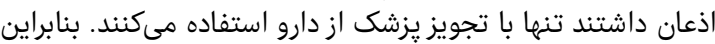

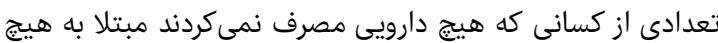

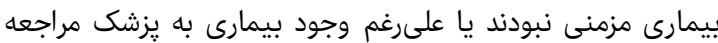

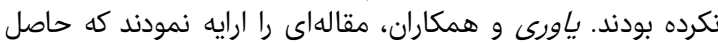

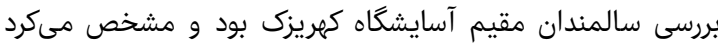

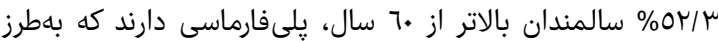

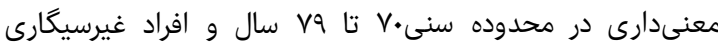

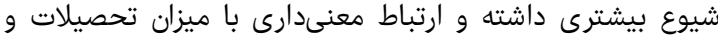

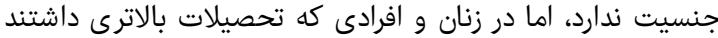

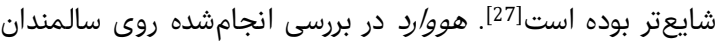

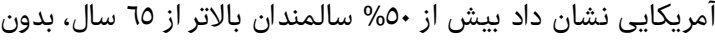

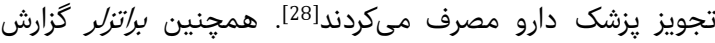

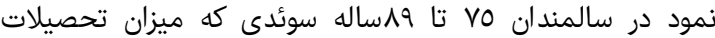

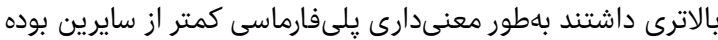

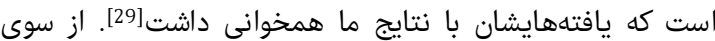

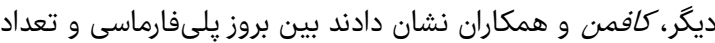

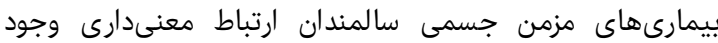

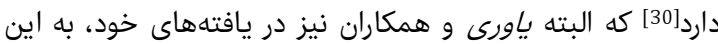

موضوع صحه كذاشته بودند [27].

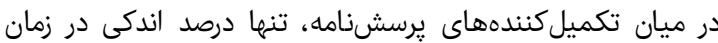

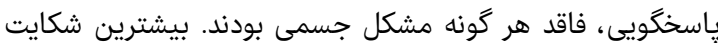

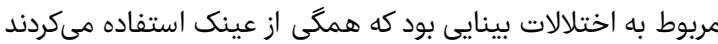

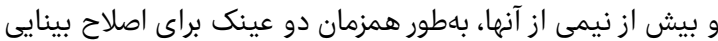

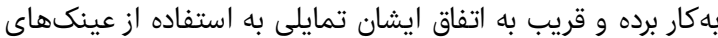

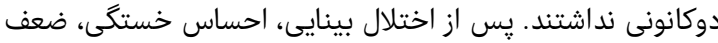

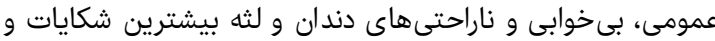


هو فراوانى بيمارىها و مشكلات جسمى در بازنشستكان نيروهاى مسلح؛ مطالعه موردى شهر تهران إندان

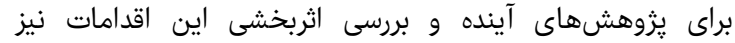
امكانيذير باشد.

\section{نتيجهاكيرى}

مهمترين مشكل سلامتى بازنشستخان نيروهاى مسلح، ابتلا به فيه

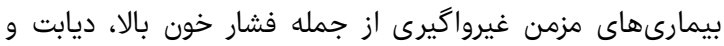

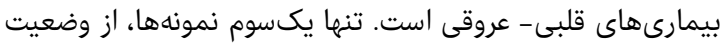

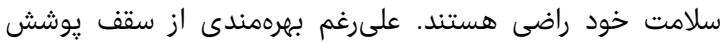

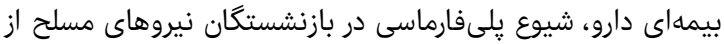

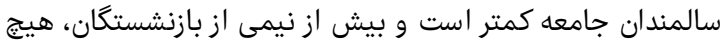
مشكل محدودكننده ارتباطى ندارند.

تشكر و قدردانى: از كليه بازنشستگانى كه منت نهاده و صبورانه به

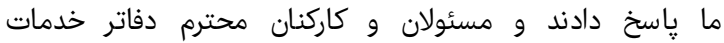

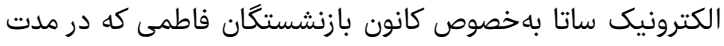

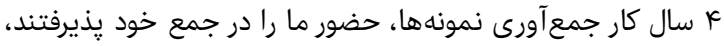
نهايت تشكر و قدردانى را داريم.

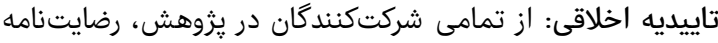

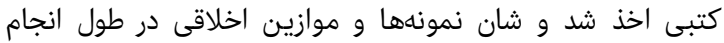

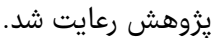
تعارض منافع: موردى از سوى نويسندكان بيان نشده است.

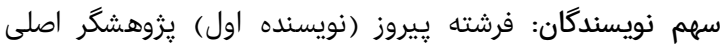

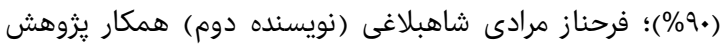

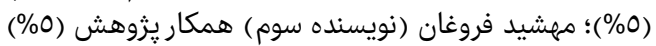

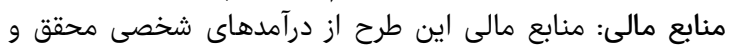

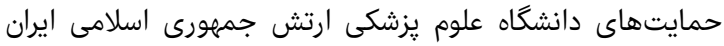
بوده است.

\section{منابع}

1- Malek Afzali H, Eftekhary Baradaran M, Hejazi F, Tabriz R, Faridi T. Community mobilization to promote the health of the elderly in the neighborhood Complex Tehran. Hakim. 2006;9(4):1-6. [Persian]

2- Harward School of Public Health. The global economic burden of non-communicable diseases in 2010. Boston: Word Economic Forum \& Harward School of Public Health; 2011. Pp. 7-37.

3- Ghasemi H, Harirchi M, Masnavi A, Rahgozar M, Akbarian M. Comparing quality of life between seniors living in families and institutionalized in nursing homes. Soc Welf. 2011;10(39):177-200. [Persian]

4- Vahdaninia M, Goshtasebi A, Montazeri A, Maftoon F. Health-related quality of life in an elderly population in Iran: A population-based study. Payesh. 2005;4(2):11320. [Persian]

5- Mirzaei M, Shams-Ghahfarkhi M. Demographic characteristics of the elderly population in Iran according to the census 1976-2006. Iran J Ageing. 2007;2(5):326-31. [Persian]

6- Mohaghghegh Kamal SH, Sajadi H, Zare H, Biglarian A. Assess the health needs aged pensioners of social security organization and national retirement fund research the city of Q om. Iran J Ageing. 2008;3(1):15-8. [Persian]

7- Xie BO. Civic Engagement among older Chinese internet users. J Appl Gerontol. 2006;2008;28(4):1-8.

8- Engel, R., Gallagher, L., \& Lyle, D. S. (2010). Military deployments and children's academic achievement: Evidence from department of defense education activity
مناسب اين بيمه در قياس با ساير سازمانهاى بيمهُ داخلى، از

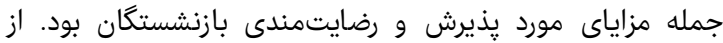

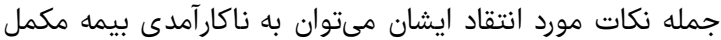

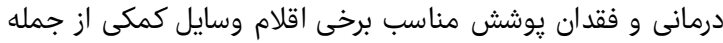

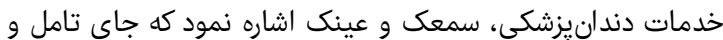

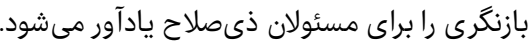

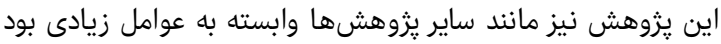

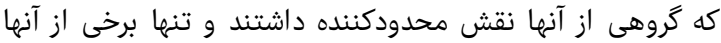

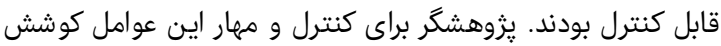

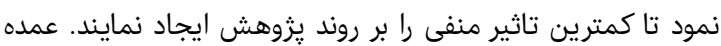

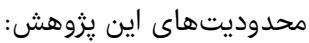

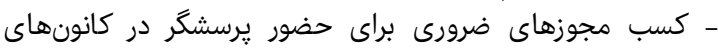

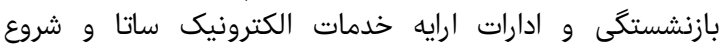

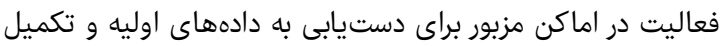

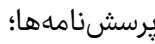
- تغيير روش و نحوهاء توام ارايه خدمات به بازنشستكان نيروهاى مسلح

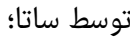

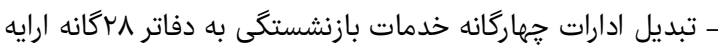

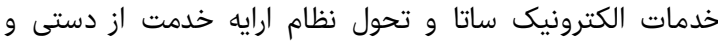

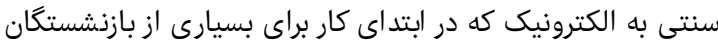
دشوار و نامانوس بودئ

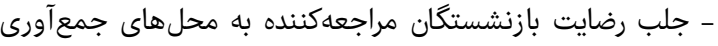

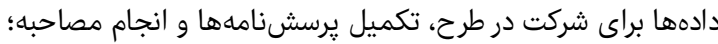

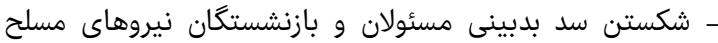

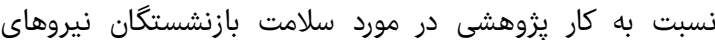

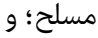
نامناسب مبودن مكان انجام مصاحبه؛

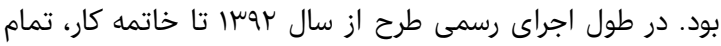

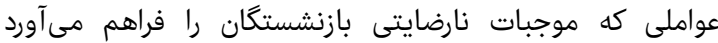

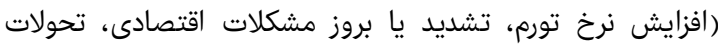

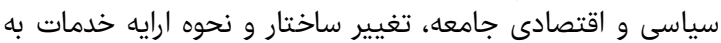

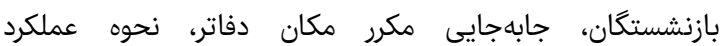

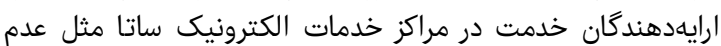

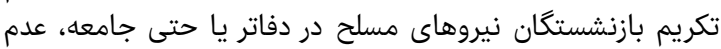

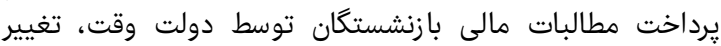

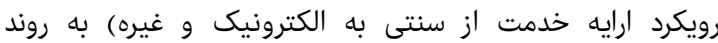

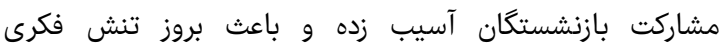

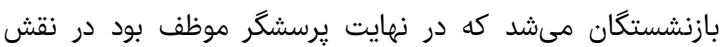

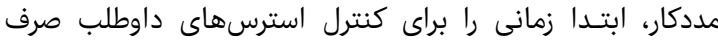

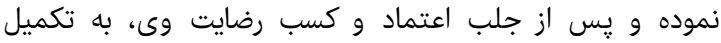

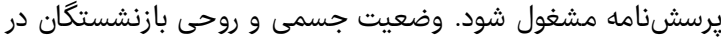

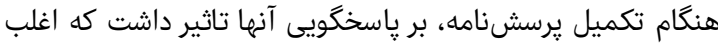

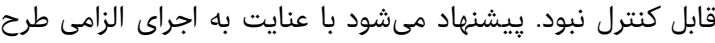

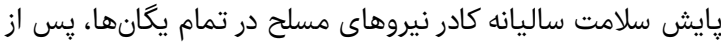

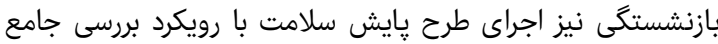

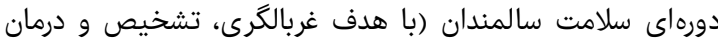

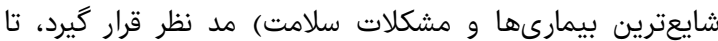

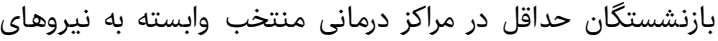

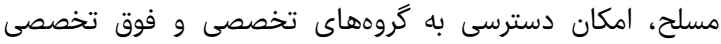

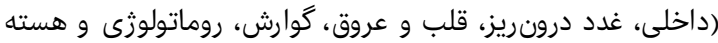

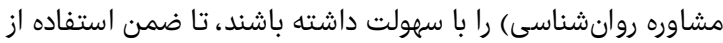

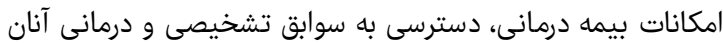


residents of Kahrizak charity foundation. Iran J Diabetes Metab. 2009;13(6):487-94. [Persian]

20- Yazdani A, Fekrazad H, Sajadi H, Salehi M. Relationship between social participation and general health among the elderly. J Kermanshah Univ Med Sci. 2010;18(10):599-606. [Persian]

21- Barati M. Fathi Y, Soltanian AR, Moeini B. Mental health condition and health promoting behaviors among elders in Hamadan. Sci J Hamadan Nurs Midwifery Fac. 2012;20(3):12-22. [Persian]

22- Nejati V. Assessing the health status of elderly people in the province of Qom (2007). J Qazvin Univ Med Sci 2009;13(1):67-72. [Persian]

23- Dandeker C, Wessely S, Iversen A, Ross J. What's in a name? defining and caring for "veterans". Armed Forces Soc. 2006;32(2):161-77.

24- Albou Korki M, Ramezani MA, Arizi F. A study on the quality of life among elderly shahinshahr area of isfahan province in year 2004. Jundishapur Sci Med J. 2007;5(4): 701-7. [Persian]

25- Noroozian M. The elderly population in Iran: An ever-growing concern in the health system. Iran J Psychiatry Behav Sci. 2012;6(2):1-6.

26- De Leo D, Diekstra RF, Lonnqvist J, Trabucchi M, Cleiren MH, Frisoni GB, et al. LEIPAD, an internationally applicable instrument to assess quality of life in the elderly. Behav Med. 1998;24(1):17-27.

27- Yavari HR, Akbari Kamrani AA, Bakhshi E, Saboor M, Sahhaf R. Association between polypharmacy and sociodemographic factors among elderly residents of kahrizak charity foundation, Tehran, 2010-2011. Salmand. 2014;8(4): 7-14. [Persian]

28- Howard RT. Inappropriate prescribing in older people. Age Ageing. 2004;33(6):530-2

29- Shalini J. Study of polypharmacy and associated problems among elderly patients. Internet J Med Update. 2012;7(1):1-10

30- Kaufman DW, Kelly JP, Rosenberg L, Anderson TE, Mitchell AA. Recent patterns of medication use in the ambulatory adult population of the United States: the Slone survey. JAMA. 2002;287(3):337-44.

31- Söderhamn O, Lindencrona C, Gustavsson SM Attitudes toward older people among nursing students and registered nurses in Sweden. Nurs Educ Today. 2001;21(3):225-9. schools. Econ Educ Rev. 2010:29(1):73-82.

9- Woodhead C, Rona RJ, Iversen AC, MacManus D, Hotopf M, Dean K, et al. Health of national Service Veterans: an analysis of a community-based sample using data from the 2007 Adult Psychiatric Morbidity Survey of England. Soc Psychiatry Psychiatr Epidemiol. 2011;46(7):559-66.

10- Hemingway H, Stafford M, Stansfeld S, Shipley M, Marmot M. Is the SF-36 a valid measure of change in population health? results from the whitehall II study. Br Med J. 1997;315(7118):1273-9.

11- Brown CS, Baker TA, Mingo CA, Harden JT, Whitfield $\mathrm{K}$, Aiken-Morgan AT, et al. A review of our roots: Blacks in gerontology, Oxford University. Gerontologist. 2014;54(1):108-16.

12- Greenberg N, Jones E, Jones N, Fear NT, Wessely S. The injured mind in the UK Armed Forces. Philos Trans R Soc Lond B Biol Sci. 2011;366(1562):261-7.

13- Rantakokko M, Portegijs E, Viljanen A, Iwarsson S, Rantanen T. Life-space mobility and quality of life in community-dwelling older people. Qual Life Res. 2016;25(5):1189-97

14- Norouzi K, Abedi H A, Maddah SSB, Mohammadi X. Development of a community based care system model for Senior Citizens in Tehran. Iran Rehabil J. 2005;3(3):48-60.

15- Mohaqeqi-Kamal H, Sajadi H, Zare H, Beiglarian A. Elderly quality of life: A comparison between pensioners of Social Security Organization and National Retirement Fund (Qom County, 2006). J Health Adm. 2007;10(27):49-57. [Persian]

16- Higate PR. Tough bodies and rough sleeping: Embodying homelessness amongst ex-servicemen. Hous Theory Soc. 2000;17(3):97-108.

17- Iversen A1, Waterdrinker A, Fear N, Greenberg N, Barker C, Hotopf M, et al. Factors associated with heavy alcohol consumption in the UK Armed Forces: Data from a health survey of Gulf, Bosnia, and Era Veterans. Mil Med. 2007;172(9):956-61.

18- Maddah SB. The status of social and leisure time activities among elderly residing in Iran and Sweden. Iran J Ageing. 2008;3(2):597-606. [Persian]

19- Bakhtiyari F, Foroughan M, Fakhrzadeh $\mathrm{H}$, Nazari N Najafi B, Alizade $M$, at al. Validation of the Persian version of abbreviated mental test (AMT) in elderly 